\title{
Treatment of Neuropathic Pain
}

\author{
Kristen Jefferies, Pharm.D. ${ }^{1}$
}

\begin{abstract}
Neuropathic pain is a common symptom associated with peripheral neuropathy and can be as or more disabling than the effects of nerve damage from the neuropathy. Though treatment of the underlying pathophysiology causing neuropathies may not be possible, treatment of neuropathic pain is. The author reviews the major medications used, dosing schedules, and data from randomized controlled trials.
\end{abstract}

KEYWORDS: Neuropathic pain, tricyclic antidepressants, selective serotonin reuptake inhibitors

Neuropathic pain is a common symptom expressed by patients who have a variety of causes for their neuropathy. It is thought to be due to pathologic changes in, or damage to, neurons in the peripheral or central nervous system. ${ }^{1}$ This disrupts the normal pain signaling process and can cause sensitization or spontaneous neuronal activity in the nervous system. The neural activity is perceived as pain. There are effective medications available for the treatment of neuropathic pain; however, many patients do not achieve a satisfactory response or experience intolerable side effects. Several organizations have published guidelines for the pharmacologic management of neuropathic pain. ${ }^{2-5}$ These guidelines are fairly consistent and emphasize the importance of medication efficacy, patient comorbidities, potential side effects and drug interactions, abuse potential, and cost when considering a medication for the treatment of neuropathic pain. The data for a large number of drugs used to treat neuropathic pain are reviewed and suggestions to optimize patient use and effect are provided. Several drugs will be discussed for off-label indications. See Table 1 for a summary of medications commonly used in the treatment of neuropathic pain.

\section{TRICYCLIC ANTIDEPRESSANTS}

Tricyclic antidepressants (TCAs) inhibit presynaptic reuptake of serotonin and norepinephrine and block cholinergic, adrenergic, histaminergic, and sodium channels. ${ }^{3}$ Several TCAs, including amitriptyline, nortriptyline, desipramine, and imipramine, have been studied for the treatment of neuropathic pain. ${ }^{6}$ This class of medications has shown positive results in the treatment of painful diabetic neuropathy, postherpetic neuralgia (PHN), painful polyneuropathy, and postmastectomy pain. Efficacy was shown in patients with and without comorbid depression. However, not all types of neuropathic pain respond to TCAs. Trials in phantom limb pain, neuropathic cancer pain, chronic lumbar root pain, chemotherapy-induced neuropathy, and human immunodeficiency virus (HIV) neuropathy had negative results. ${ }^{6}$

The Neuropathic Pain Special Interest Group (NeuPSIG) of the International Association for the Study of Pain (IASP) recommends secondary amine TCAs (nortriptyline and desipramine) as first-line treatment for neuropathic pain and tertiary amines (amitriptyline and imipramine) if a secondary amine is not available. ${ }^{5}$ The secondary amines are typically better

\footnotetext{
${ }^{1}$ Department of Pharmacy Services, University of Utah Hospital, Salt Lake City, Utah.

Address for correspondence and reprint requests: Kristen Jefferies, Pharm.D., Department of Pharmacy Services, University of Utah Hospital, 50 N. Medical Drive, Room A-050, Salt Lake City, UT 84132 (e-mail: Kristen.Jefferies@hsc.utah.edu).

Peripheral Neuropathy; Guest Editor, Mark B. Bromberg,
}

M.D., Ph.D.

Semin Neurol 2010;30:425-432. Copyright (C) 2010 by Thieme Medical Publishers, Inc., 333 Seventh Avenue, New York, NY 10001, USA. Tel: +1(212) 584-4662.

DOI: http://dx.doi.org/10.1055/s-0030-1267286.

ISSN 0271-8235. 

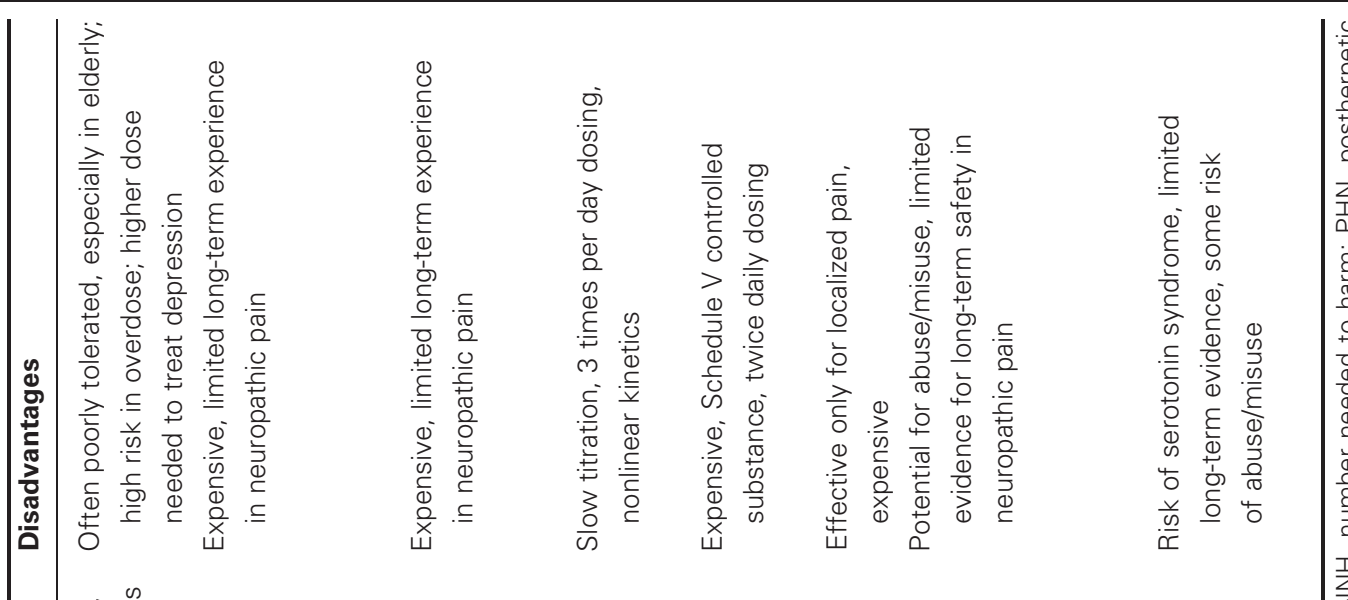

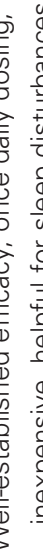
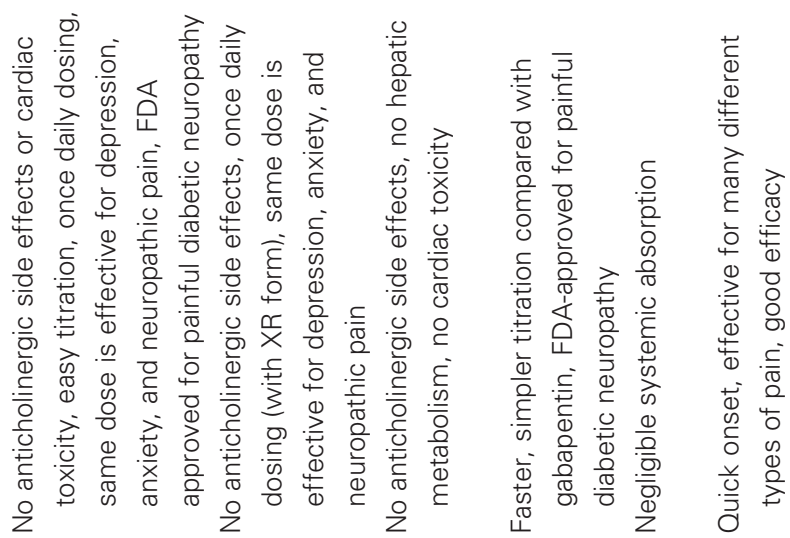

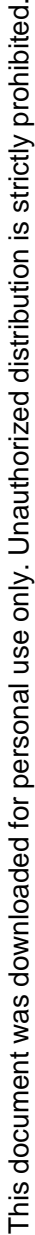

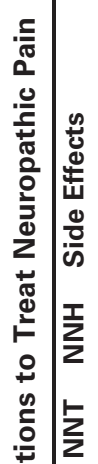

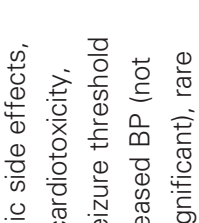
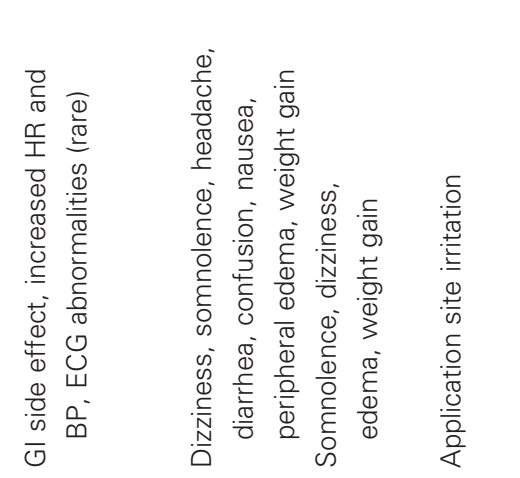

$-\quad 0 . \frac{\pi}{0}$
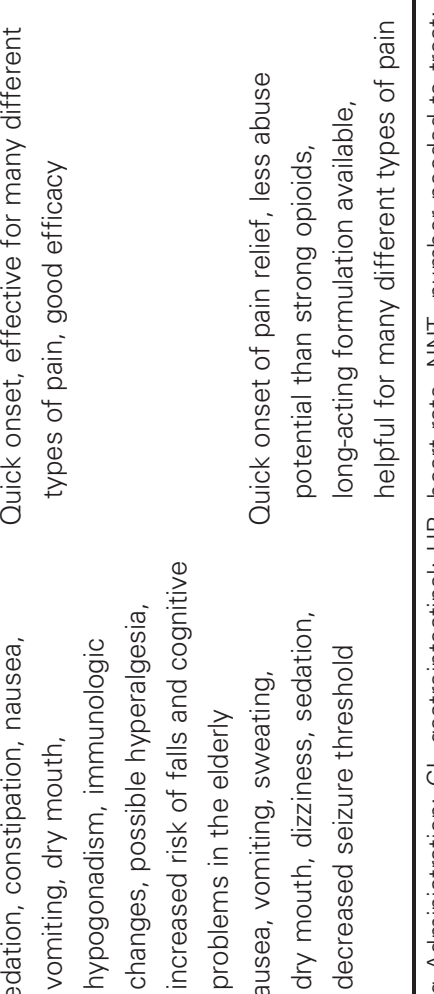

$\infty$

$\stackrel{\varphi}{0}$

s

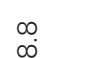

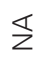

$\bar{m} \quad \stackrel{m}{+}$

เ $\quad \mathbb{z}$

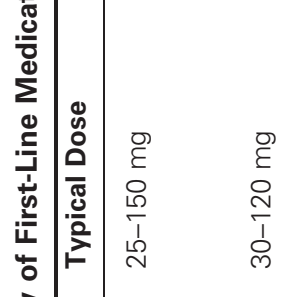

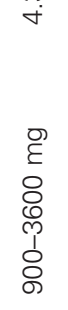

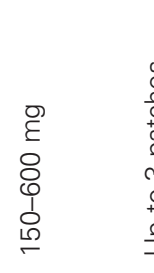

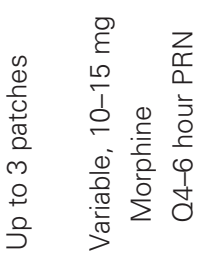

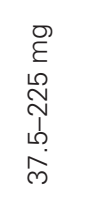

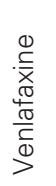

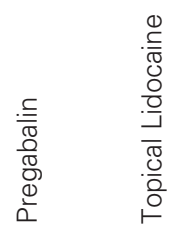
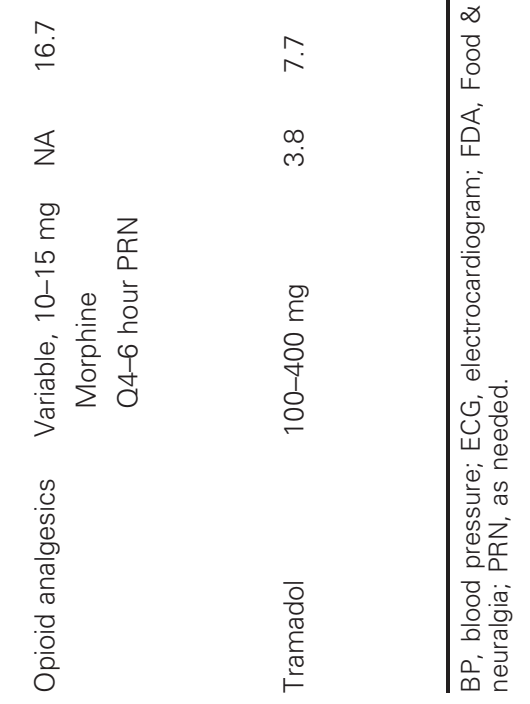
tolerated with similar efficacy. The European Federation of Neurological Societies (EFNS) task force recommends TCAs as first-line treatment for painful polyneuropathy, postherpetic neuralgia, and central neuropathic pain. ${ }^{2}$ The Canadian Pain Society (CPS) recommends TCAs as first-line treatment for neuropathic pain due to diabetes, herpes zoster, and traumatic nerve injury or stroke. ${ }^{4}$

The typical starting dose of any TCA for the treatment of neuropathic pain is 10 to $25 \mathrm{mg}$ nightly. This can be increased by 10 to $25 \mathrm{mg}$ every 3 to 7 days as tolerated up to a usual maximum of $150 \mathrm{mg}$ nightly. A trial of 6 to 8 weeks at the maximum tolerated dose is necessary to assess the benefit of the medication. ${ }^{6}$ It may take several weeks to see improvement in pain.

The advantages of TCAs include their effect on common comorbidities such as insomnia and depression. They are inexpensive and dosed once daily. ${ }^{6}$ Anticholinergic side effects occur frequently and may be dose limiting. These include dry mouth and eyes, urinary retention, excess sedation, orthostatic hypotension, constipation, and blurry vision. Starting at a lower dose and titrating very slowly may reduce these side effects. ${ }^{6}$ Rare, but serious side effects include decreased seizure threshold and cardiac toxicity. Due to reports of tachycardia, myocardial infarction, and sudden cardiac death (at doses greater than $100 \mathrm{mg}$ per day) the NeuPSIG recommends a baseline echocardiogram for patients over 40 years of age and avoiding TCAs in patients at risk for sudden cardiac death or with a history of cardiovascular disease. ${ }^{5}$ TCAs have a high risk of mortality in an overdose and should be avoided in patients at risk for suicide attempts. ${ }^{6}$ In elderly patients, TCAs can exacerbate cognitive problems and increase the risk of falls. ${ }^{5}$

A Cochrane review ${ }^{7}$ of 13 placebo controlled trials that included patients with a variety of neuropathic pain conditions concluded that TCAs were more effective than placebo. From an additional 12 studies that compared one TCA to another, there was no significant difference in efficacy among TCAs. The number needed to treat $(\mathrm{NNT})$ to achieve moderate pain relief was 3.6 (95\% CI 3-4.5) for all TCAs, 3.1 (95\% CI 2.5-4.2) for amitriptyline $<150 \mathrm{mg}, 2.6$ (95\% CI 1.9-4.5) for desipramine, and 2.2 (95\% CI 1.7-3.2) for imipramine. The number needed to harm (NNH), defined as causing side effects that led to discontinuation of the medications, was 28 (95\% CI 17-68).

\section{SEROTONIN-NOREPINEPHRINE REUPTAKE INHIBITORS (SNRIs)}

Duloxetine (Cymbalta ${ }^{\circledR}$; Eli Lilly \& Co., Indianapolis, IN) and venlafaxine (Effexor ${ }^{\circledR}$; Pfizer Pharmaceuticals, New York, NY) are the two serotonin-norepinephrine inhibitors studied for the treatment of neuropathic pain. Milnaciprin $\left(\right.$ Savella ${ }^{\mathbb{R}}$; Forest Pharmaceuticals, New York, NY) is another SNRI that is effective for fibromyalgia, but has not been studied for neuropathic pain. The EFNS and NeuPSIG recommend SNRIs as firstline options for the treatment of painful diabetic neuropathy, ${ }^{2,5}$ and the Canadian Pain Society recommends this class of medications as second-line treatment options. ${ }^{4}$ However, Canadian guidelines were published prior to the completion of the more recent studies of SNRIs.

\section{Duloxetine (Cymbalta ${ }^{\circledR}$ )}

Duloxetine is approved by the Food and Drug Administration (FDA) for painful diabetic neuropathy as well as major depressive disorder, generalized anxiety disorder, and fibromyalgia. ${ }^{8}$ It has not been studied for other types of neuropathic pain. ${ }^{6}$

The typical starting dose of duloxetine is 20 to $30 \mathrm{mg}$ daily, which can be increased in $20 \mathrm{mg}$ or 30 $\mathrm{mg}$ increments every week up to $60 \mathrm{mg}$ daily. Initial treatment at $60 \mathrm{mg}$ daily is associated with more nausea. Doses higher than $60 \mathrm{mg}$ daily have not consistently shown additional benefit in clinical trials. A trial of 4 weeks at the maximum dose is considered adequate. $^{6}$

Long duration studies are available for duloxetine. One open-label extension study showed continued benefit with duloxetine lasting up to 1 year. ${ }^{6}$ The same dose is effective for treating depression, anxiety, and neuropathic pain, which makes duloxetine useful in patients with these comorbidities. ${ }^{6}$ Duloxetine can be titrated to an effective dose in only one week. Duloxetine is typically well tolerated and is not associated with the anticholinergic side effects that often limit the use of TCAs. However, nausea is a common side effect of duloxetine. This typically improves over time and is less likely when the drug is initiated at a lower dose $(30 \mathrm{mg}$ daily). Other side effects include hepatotoxicity and increased blood pressure and heart rate, which are not typically clinically significant. ${ }^{6}$ Duloxetine is not yet available as a generic and is much more expensive than the TCAs.

A Cochrane review ${ }^{9}$ assessed the efficacy of duloxetine for neuropathic pain. This review included three trials (1139 patients) comparing duloxetine to placebo for treatment of painful diabetic neuropathy. The NNT with duloxetine $60 \mathrm{mg}$ daily for 3 months to achieve over $50 \%$ improvement in pain was 6 (95\% CI 5-10). To achieve over 30\% improvement in pain, the NNT was 5 (95\% CI 3-8). Each of the three duloxetine trials had dropout rates of over $20 \%$ with $\sim 16 \%$ of patients discontinuing due to side effects. The $\mathrm{NNH}$ for duloxetine was 17 (95\% CI 12-50) for side effects leading to discontinuation of medication. 


\section{Venlafaxine}

Venlafaxine is available in an immediate release formulation dosed three times daily and extended release formulation dosed once daily. It is approved by the FDA for the treatment of major depressive disorder, generalized anxiety disorder, panic disorder, and social anxiety disorder. ${ }^{10}$

The initial dose is $37.5 \mathrm{mg}$ daily or twice daily and can be titrated by 37.5 to $75 \mathrm{mg}$ each week as tolerated. The maximum dose is $225 \mathrm{mg}$ per day. A trial of 4 to 6 weeks at a dose of at least $150 \mathrm{mg}$ per day is necessary to assess the benefit of venlafaxine. ${ }^{6}$ Like duloxetine, a relatively fast titration is possible with venlafaxine, with the ability to reach an effective dose for both pain and depression in 2 to 3 weeks. ${ }^{6}$

Electrocardiogram abnormalities were reported in $5 \%$ of patients treated with venlafaxine in one clinical trial. ${ }^{5}$ Elevated blood pressure and heart rate have also been reported. Gastrointestinal side effects are the most common side effects.

Venlafaxine was superior to placebo for the treatment of painful diabetic neuropathy and painful polyneuropathies of other etiologies at doses of 150 to 225 mg per day. ${ }^{6}$ It was no better than placebo for the treatment of $\mathrm{PHN}$ or postmastectomy pain; however, some of these studies used doses less than $150 \mathrm{mg}$ daily. ${ }^{6}$ The efficacy of venlafaxine for neuropathic pain was assessed in a Cochrane review. ${ }^{7}$ This review included three studies of venlafaxine (extended release formulation) at doses of 75 to $225 \mathrm{mg}$ daily. The NNT to achieve moderate pain relief was 3.1 (95\% CI 2.2-5.1). The $\mathrm{NNH}$ for side effects leading to discontinuation of the medication was 16 (95\% CI 8-436).

\section{CALCIUM CHANNEL ALPHA-2-DELTA LIGANDS}

Gabapentin (Neurontin ${ }^{\circledR}$, Pfizer Pharmaceuticals) and pregabalin (Lyrica ${ }^{\circledR}$, Pfizer Pharmaceuticals) are structurally similar to gamma-aminobutyric acid (GABA), although they do not bind to GABA receptors. They are thought to exert their beneficial effects on neuropathic pain by binding to the $\alpha$-2-delta subunit of voltage-dependant calcium channels. This leads to reduction of the influx of calcium into neurons throughout the central nervous system (CNS). ${ }^{3}$ This in turn may decrease the release of glutamate, norepinephrine, and substance P. ${ }^{5}$

\section{Gabapentin (Neurontin ${ }^{\circledR}$ )}

Gabapentin is an antiepileptic drug (AED) studied for various types of neuropathic pain. It is FDA-approved for the treatment of PHN and as adjunct therapy for partial onset seizures. ${ }^{11}$ Clinical trials have shown positive results for the treatment of PHN and painful polyneuropathy, and mixed results for the treatment of painful diabetic neuropathy and phantom limb pain. Gabapentin was not effective in studies of complex regional pain syndrome, chemotherapy-induced neuropathy, and HIV neuropathy. ${ }^{6}$ Gabapentin is recommended as a first-line treatment option for painful polyneuropathies, postherpetic neuralgia, and central neuropathic pain by the AISP, EFNS, and CPS. ${ }^{2,4,5}$

Gabapentin can be initiated at doses of 100 to 300 $\mathrm{mg}$ at bedtime or 100 to $300 \mathrm{mg}$ three times daily. The dose should be titrated by 100 to $300 \mathrm{mg}$ every 3 to 7 days as tolerated to a maximum dose of $3600 \mathrm{mg}$ per day. The usual effective dose is $1800 \mathrm{mg}$ to $3600 \mathrm{mg}$ per day, which may take several weeks to achieve. Because of the slow dose titration, an adequate trial may take more than 2 months. ${ }^{5}$ It has minimal drug interactions because it is not hepatically metabolized and does not inhibit or induce hepatic enzymes. However, a dose reduction is required in patients with renal insufficiency. It may also help improve sleep.

Sedation is the most common dose-limiting side effect and is minimized by initiating with a lower dose and titrating more gradually. ${ }^{6}$ Somnolence and dizziness are the most common side effects, and can often be managed by a slow titration. The elderly are more prone to these side effects and gabapentin may increase the risk of falls and worsen cognitive impairment in this patient population. ${ }^{5}$ The slow-dose titration and onset of action, three times daily dosing schedule and side effects of sedation, edema, dizziness, and weight gain may limit the use of gabapentin for some patients.

A Cochrane review ${ }^{12}$ included trials of gabapentin for the treatment of different types of chronic pain. This analysis included four placebo-controlled trials (281 patients) of gabapentin 900 to $3600 \mathrm{mg}$ daily for the treatment of painful diabetic neuropathy. The combined NNT for effective pain relief in these four studies was 4.3 (95\% CI 3.5-5.7); that is, $64 \%$ of patients improved on gabapentin compared with $28 \%$ on placebo. Three control studies comparing gabapentin to amitriptyline for the treatment of painful diabetic neuropathy were also reported. One study of 25 patients showed similar efficacy with gabapentin (900-1800 mg per day) or amitriptyline (25-75 $\mathrm{mg}$ per day). A second study of 25 patients concluded that gabapentin (1200-2400 mg per day) was superior to amitriptyline (30-90 mg daily), but these results were not statistically significant. The third study included only seven patients who had benefit from gabapentin in a previous study. The results of this study were not evaluable. ${ }^{12}$

A recent crossover design study ${ }^{13}$ compared nortriptyline (maximum dose of $100 \mathrm{mg}$ daily), gabapentin (maximum dose of $3600 \mathrm{mg}$ daily), and a combination of both in 56 patients with painful diabetic neuropathy or PHN. Participants received each treatment for 6 weeks 
followed by a one week taper and a one-week washout phase. Pain scores were significantly lower during the combination phase than for either treatment alone. Gabapentin monotherapy and nortriptyline monotherapy were similarly effective. Dry mouth was more common with nortriptyline and difficulty concentrating was more common with gabapentin.

\section{Pregabalin (Lyrica ${ }^{\circledR}$ )}

Pregabalin is thought to have a mechanism of action similar to gabapentin. It is FDA-approved for the treatment of painful diabetic neuropathy, PHN, and fibromyalgia and as adjunct therapy for partial onset seizures. Its efficacy is established in randomized controlled trials for the treatment of painful diabetic neuropathy and PHN; however, some trials in these conditions have also shown negative results. ${ }^{6}$

Pregabalin is initiated at a dose of $50 \mathrm{mg}$ three times daily or $75 \mathrm{mg}$ twice daily. The total daily dose can be titrated in increments of $150 \mathrm{mg}$ every 3 to 7 days as tolerated to a maximum dose of $600 \mathrm{mg}$ per day. However, doses greater than $300 \mathrm{mg}$ have not consistently shown additional benefit for the treatment of neuropathic pain conditions. ${ }^{6}$ A lower initial dose and slower taper may help minimize sedation. Pregabalin has a faster tolerable titration than does gabapentin and twice daily rather than three times per day dosing. Pregabalin has minimal drug interactions and no hepatic metabolism. It can help with comorbidities such as insomnia and anxiety. ${ }^{6}$

The side effects associated with pregabalin also appear to be similar to those associated with gabapentin, including sedation, edema, dizziness, and weight gain and occur more frequently at higher doses. A small percentage of patients reported euphoria when taking pregabalin, leading to its Schedule V Controlled Substance classification in the United States. ${ }^{6}$

A Cochrane review assessed the efficacy of pregabalin for chronic pain. The NNT for greater than 50\% pain relief over baseline with $600 \mathrm{mg} /$ day was $5.0(95 \%$ CI 4.0-6.6) for painful diabetic neuropathy (six studies, 1360 patients) and 5.6 (95\% CI 3.5-14) for central neuropathic pain (two studies, 176 patients). The NNT for greater than $50 \%$ pain reduction at a lower dose of $300 \mathrm{mg}$ per day was 7.5 (95\% CI 5.1-14) for painful diabetic neuropathy (two studies, 341 patients). The results were similar when only studies of greater than 8-week duration were included. The $\mathrm{NNH}$ causing discontinuation due to side effects with $600 \mathrm{mg} /$ day was 8.8 (95\% CI 6.8-12) in painful diabetic neuropathy trials (six studies, 1351 patients), and not significantly different from placebo in central neuropathic pain trials. For a lower dose of $300 \mathrm{mg}$ per day, the NNH was 16 (95\% CI 9.9 to 37) for painful diabetic neuropathy (four studies, 823 patients).

\section{TOPICAL LIDOCAINE}

Topical lidocaine is thought to reduce discharges of small afferent nerve fibers by blocking voltage-gated sodium channels. ${ }^{3}$ It is available in gel and transdermal patch formulations. The transdermal patch is FDA approved for treatment of PHN. ${ }^{14}$

Lidocaine 5\% transdermal patch was effective in randomized controlled trials of patients with allodynia due to PHN or peripheral neuropathies of other etiologies. ${ }^{6}$ A lidocaine gel formulation was effective in patients with PHN and allodynia, but can also be considered when the transdermal patch is not available, is not tolerated, or is too expensive. ${ }^{5}$ NeuPSIG recommends topical lidocaine as a first-line option for the treatment of localized peripheral neuropathic pain, ${ }^{5}$ and the EFNS recommends it as first-line treatment of PHN with allodynia. Topical lidocaine is considered a secondline treatment option for localized neuropathic pain by the CPS. ${ }^{4}$

A maximum of three patches can be applied to the painful area once every 24 hours and left in place for 12 hours. Prior to removing the release liner, the patches can be cut to fit the affected area. ${ }^{14}$ Topical lidocaine is well tolerated and minimal systemic absorption occurs at the recommended dose. ${ }^{5}$ No dose titration is necessary to reach an effective dose. ${ }^{6}$

Lidocaine patches are effective only for the area where the patch is applied, and are not helpful for central neuropathic pain or to treat polyneuropathy that occurs over a large area. ${ }^{6}$ Application site irritation may occur. Although systemic absorption is unlikely, topical lidocaine should be used with caution in patients with hepatic dysfunction, and those taking class I antiarrhythmic medications such as mexiletine. ${ }^{5}$ Increased absorption or drug accumulation may occur when the patch is applied for longer periods of time or over larger areas than recommended, in small patients, and in patients with kidney insufficiency. ${ }^{14}$

\section{OPIOID ANALGESICS}

Randomized controlled trials have shown beneficial effects of opioids (including oxycodone, methadone, morphine, and levorphanol) for the treatment of DPN, PHN, painful polyneuropathy, and phantom limb pain. ${ }^{6}$ Comparative trials have shown similar benefit with opioids when compared with TCAs and gabapentin, but more frequent side effects occurred with opioids. ${ }^{6}$ NeuPSIG recommends opioids as second-line medications when an adequate response is not achieved with first-line medications or as a firstline option when immediate pain relief is necessary and short-term for acute neuropathic pain. ${ }^{5}$ The EFNS recommends opioid medications as second- or third-line options for painful diabetic neuropathy, PHN, and central neuropathic pain because of limited 
trials assessing long-term safety and abuse potential. ${ }^{2}$ The Canadian Pain Society recommends opioids (excluding methadone) as third-line agents for the treatment of painful diabetic neuropathy, PHN, and other neuropathic pain conditions. Methadone is considered a fourth-line agent because of limited clinical evidence, difficulty with titration, and regulatory status in Canada. ${ }^{4}$

The appropriate dose is widely variable from patient to patient and depends on prior opioid exposure. The typical initial dose is 10 to $15 \mathrm{mg}$ of morphine or equianalgesic dose every 4 hours or as needed. After 1 to 2 weeks the total daily dose can be converted to an equianalgesic dose of a longer acting opioid medication such as transdermal fentanyl, extended release oxycodone, or extended release morphine. A scheduled longacting opioid is typically preferred. If pain is not improved after an adequate trial, the medication should be tapered and discontinued. ${ }^{5}$

Compared with other medications, opioids typically have a quick onset of pain relief. Opioid medications are particularly useful for short-term use to treat acute exacerbations or during the titration phase of a first-line medication. ${ }^{5}$ There are several disadvantages associated with the use of opioid medications. They are often poorly tolerated. Common side effects include sedation, constipation, and nausea. Constipation typically does not improve over time and a bowel regimen may be required. These medications can increase risk of falls and cognitive problems in elderly patients. Rare but serious side effects include hypogonadism, immunologic changes, and hyperalgesia. Opioids are associated with a risk of misuse or addiction of $5 \%$ to $50 \%$ in other chronic pain conditions. Risk factors for abuse include a history of or current substance abuse, major psychiatric disorders, and family history of substance abuse. $^{5}$

A Cochrane review of 23 studies assessed the efficacy of opioid medications for the treatment of neuropathic pain. ${ }^{15}$ The majority of these studies assessed treatment for less than 24-hour duration, single doses, or intravenous (IV) administration, but nine studies were 8 to 70 days in duration and included 460 patients with various types of neuropathic pain (painful diabetic neuropathy, PHN, and phantom limb pain). The opioid medications studied were morphine (four trials), oxycodone (three trials), methadone (two trials), and levorphanol (one trial). The opioid medications were superior to placebo in all placebo-controlled studies. A meta-analysis of the seven studies with suitable data concluded that overall opioid medications were associated with a reduction in pain intensity of 13 points on a scale of 0 to 100 . The most common side effects were nausea, constipation, sedation, and vomiting. The NNH for drug discontinuation was 16.7 (95\% CI 9.1-100).

\section{TRAMADOL (ULTRAM ${ }^{\circledR}$ )}

Tramadol (Ultram ${ }^{\circledR}$; Ortho-McNeil-Jansson Pharmaceuticals, Titusville, NJ) is a weak Mu opioid receptor agonist and weakly inhibits serotonin and norepinephrine reuptake. Clinical trials have shown efficacy for tramadol in the treatment of painful diabetic neuropathy, PHN, painful polyneuropathy, and phantom limb pain. ${ }^{6}$ The NeuPSIG recommends tramadol as a second-line treatment for neuropathic pain but may be used as first-line treatment in the same clinical situations when opioids could be considered first line. ${ }^{5}$ The EFNS recommends tramadol as a second- or third-line treatment option for painful diabetic neuropathy, PHN, and central neuropathic pain and as a first-line option for acute exacerbation of painful diabetic neuropathy. ${ }^{2} \mathrm{Tra}-$ madol is recommended as a third-line agent by the CPS. $^{4}$

Tramadol is typically initiated at a dose of $50 \mathrm{mg}$ one to two times per day and titrated as needed to a maximum total daily dose of $400 \mathrm{mg}(100 \mathrm{mg} 4$ times daily). It is available in immediate release and sustained release formulations and in combination with acetaminophen.

Like the strong opioid analgesics, tramadol is associated with a quick onset of pain relief. It is associated with a lower risk of abuse than strong opioid receptor agonists and is less sedating. ${ }^{6}$

Common side effects of tramadol include sedation, nausea, constipation, orthostatic hypotension, and decreased seizure threshold. Tramadol may be associated with an increased risk of serotonin syndrome when combined with other serotonergic drugs. ${ }^{5}$

A Cochrane review assessed the use of tramadol for neuropathic pain. ${ }^{16}$ In five placebo controlled studies of 4 to 7 weeks duration, tramadol was superior to placebo for the treatment off various types of neuropathic pain. A meta-analysis of three of these studies (303 patients) calculated a NNT of 3.8 (95\% CI 2.8-6.3) for a $50 \%$ reduction in pain. The most common side effects were nausea, vomiting, sweating, dry mouth, dizziness, and sedation. The NNH to cause drug discontinuation was 7.7 (95\% CI 4.6-20). In these studies, tramadol was associated with a very low abuse potential.

\section{TOPICAL CAPSAICIN}

Capsaicin depletes substance P. Reviews of six studies (389 patients) of capsaicin $0.075 \%$ cream versus placebo, and two studies (709 patients) of a single application of a capsaicin $8 \%$ transdermal patch versus placebo revealed the following: The NNT with capsaicin $0.075 \%$ cream for 6 to 8 weeks was 6.6 (95\% CI 4.1-17) to achieve any pain relief. The NNT with the capsaicin patch to achieve $>30 \%$ pain reduction at 12 weeks was 12 (95\% CI 6.4 to 70). However, it was noted that the data used to calculate the NNT was not robust due to the small study sizes and 
differences in outcomes used. The most common side effects reported were local skin irritation (burning, stinging, erythema, rash, itching), which typically improved after 1 to 2 weeks. Topical capsaicin is generally recommended as a third-line treatment option when other medications are ineffective or not tolerated. Various topical formulations of low concentration capsaicin are available without a prescription in the United States. In November 2009, capsaicin $8 \%$ patch was approved by the FDA for the treatment of PHN. It is administered for 1 hour every 3 months and professional training is required. ${ }^{17}$

\section{MEDICATIONS WITH LESS EFFICACY DATA}

\section{Serotonin Reuptake Inhibitors (SSRIs)}

A Cochrane review published in $2007^{7}$ assessed the efficacy of antidepressants for the treatment of neuropathic pain. This review included three small studies that compared paroxetine $\left(\mathrm{Paxil}^{\mathbb{R}}\right)$, citalopram $\left(\mathrm{Celexa}{ }^{\mathbb{R}}\right.$; Forest Laboratories), and fluoxetine (Prozac ${ }^{\circledR}$, Eli Lilly \& Company) to placebo for the treatment of painful diabetic neuropathy. The SSRIs were superior to placebo in all studies. A comparative study of 26 patients with painful diabetic neuropathy concluded that paroxetine was superior to placebo but less effective than imipramine. Escitalopram (Lexapro ${ }^{\mathbb{R}}$; Forest Laboratories) was superior to placebo in a recent randomized, blinded, crossover study in 41 patients with painful diabetic neuropathy. ${ }^{18}$ Seven patients reported good pain relief with escitalopram compared with no patients reporting good pain relief with placebo. Bupropion (Wellbutrin ${ }^{\mathbb{R}}$; GlaxoSmithKline, Brentford, Middlesex, UK) was superior to placebo in a small, single center, randomized, placebo controlled trial in 21 patients with various types of neuropathic pain. ${ }^{19}$

\section{Other Antiepileptic Drugs}

Lamotrigine (Lamictal ${ }^{\mathbb{R}}$; GlaxoSmithKline) is FDAapproved for the treatment of bipolar disorder and as adjunctive treatment or monotherapy for various types of seizures. ${ }^{20}$ It is thought to exert its pain-relieving effect by blocking voltage-dependent sodium channels. This inhibits presynaptic release of excitatory amino acids. ${ }^{3} \mathrm{~A}$ Cochrane review ${ }^{21}$ assessed the efficacy of lamotrigine for acute and chronic pain. This review included one study of 59 patients with painful diabetic neuropathy. Twelve of 27 patients on lamotrigine reported a 50\% reduction in pain compared with 5 of 26 patients on placebo. This was not statistically significant. A more recent study also concluded lamotrigine was "inconsistently effective" compared with placebo for the treatment of painful diabetic neuropathy. ${ }^{22}$ Lamotrigine was similar to amitriptyline for the treatment of painful diabetic neuropathy in a crossover trial of 46 patients. ${ }^{23}$ However, only six patients were evaluated at the end of the first six week treatment phase and only three patients were evaluated at the end of the second phase. Lamotrigine was similar to placebo in a combination trial of 220 patients with various types of neuropathic pain. ${ }^{24}$ This study included patients whose pain was not controlled by gabapentin, TCAs, or nonopioid analgesics. The addition of lamotrigine was not associated with more benefit than placebo.

Lacosamide (Vimpat ${ }^{\circledR}$; UCB Pharma, Smyrna, GA) was recently FDA-approved for adjunct treatment of partial onset seizures. ${ }^{25}$ It has been studied for the treatment of painful diabetic neuropathy, but the FDA did not approve it for this indication. It is thought to control neuronal excitation by inactivating voltage-gated sodium channels. It may also have some beneficial effects on pain by modulating NMDA receptors. ${ }^{26}$ Clinical trials of lacosamide for painful diabetic neuropathy have mixed results, with some showing slight improvement in pain and some showing no difference from placebo.

Carbamazepine (Tegretol ${ }^{\mathbb{R}}$; Novartis Pharmaceuticals, East Hanover, NJ) is approved in the United States for the treatment of pain associated with trigeminal neuralgia and various types of seizures. Limited data exists to evaluate its role in other types of neuropathic pain. $^{27}$

Other antiepileptic drugs, including oxcarbazepine (Trileptal ${ }^{\mathbb{R}}$; Novartis Pharmaceuticals), topiramate $\left(\right.$ Topamax ${ }^{\mathbb{B}}$; Ortho-McNeil, Inc., Raritan, NJ), levetiracetam (Keppra ${ }^{\circledR}$; UCB Pharma), and valproic acid (Depakote ${ }^{\circledR}$; Abbott Laboratories, Abbott Park, IL), have limited evidence and/or inconsistent results in the treatment of neuropathic pain. $3,5,6,28,29$

\section{Miscellaneous Medications}

Intradermal botulinum toxin was superior to placebo in a single study of 29 patients with DPN. ${ }^{30}$ Studies in PHN have inconsistent results. ${ }^{29}$ Further research is needed to determine its role in neuropathic pain treatment.

Mexiletine (Mexitil ${ }^{\circledR}$; Teva Pharmaceuticals, North Wales, PA) and lidocaine are sodium channel blockers. In a metanalysis, ${ }^{31}$ intravenous lidocaine and its oral analog, mexiletine, were superior to placebo for the treatment of various types of neuropathic pain. No other randomized controlled trials of lidocaine or mexiletine in the treatment of neuropathic pain have been published.

NMDA receptor antagonists have been used for painful neuropathies. Memantine (Namenda ${ }^{\mathbb{R}}$; Forest Pharmaceuticals) is FDA-approved for the treatment of Alzheimer's dementia. Trials of memantine for the treatment of neuropathic pain have inconsistent results, including one study of painful diabetic neuropathy that 
showed it was not better than placebo. ${ }^{32}$ Dextromethorphan is another NMDA glutamate receptor antagonist with inconsistent results for the treatment of neuropathic pain. ${ }^{4}$ A combination of dextromethorphan and quinidine has been studied for pseudobulbar affect associated with multiple sclerosis and amyotrophic lateral sclerosis with promising results. Low dose quinidine inhibits the metabolism of dextromethorphan, leading to a longer half-life and higher concentrations of dextromethorphan. A single open-label study ${ }^{33}$ assessed the safety of this combination in the treatment of DPN. Dropout rates and side effects were similar to those in previous studies for pseudobulbar affect. More research is needed to assess the efficacy of dextromethorphan combined with quinidine for neuropathic pain conditions.

\section{REFERENCES}

1. Freynhagen R, Bennett MI. Diagnosis and management of neuropathic pain. BMJ 2009;339:b3002

2. Attal N, Cruccu G, Baron R, et al. EFNS guidelines on the pharmacological treatment of neuropathic pain: 2009 revision. Eur J Neurol 2010;(Apr):9

3. Jensen TS, Madsen CS, Finnerup NB. Pharmacology and treatment of neuropathic pains. Curr Opin Neurol 2009;22(5): 467-474

4. Moulin DE, Clark AJ, Gilron I, et al; Canadian Pain Society. Pharmacological management of chronic neuropathic pain consensus statement and guidelines from the Canadian Pain Society. Pain Res Manag 2007;12(1):13-21

5. Dworkin RH, O'Connor AB, Backonja M, et al. Pharmacologic management of neuropathic pain: evidence-based recommendations. Pain 2007;132(3):237-251

6. O'Connor AB, Dworkin RH. Treatment of neuropathic pain: an overview of recent guidelines. Am J Med 2009; 122(10, Suppl):S22-S32

7. Saarto T, Wiffen PJ. Antidepressants for neuropathic pain. Cochrane Database Syst Rev 2007;(4):CD005454

8. Eli Lilly and Co. Cymbalta prescribing information. Available at: http://www.cymbalta.com/index.jsp. Accessed June 30, 2010

9. Lunn MP, Hughes RA, Wiffen PJ. Duloxetine for treating painful neuropathy or chronic pain. Cochrane Database Syst Rev 2009;(4):CD007115

10. Pfizer. Effexor prescribing information. Available at: http:// www.effexor.com/. Accessed June 30, 2010

11. Pfizer. Neurontin prescribing information. Available at: http://www.pfizer.com/products/rx/rx_product_neurontin.jsp. Accessed June 30, 2010

12. Wiffen PJ, McQuay HJ, Edwards JE, Moore RA. Gabapentin for acute and chronic pain. Cochrane Database Syst Rev 2005;(3):CD005452

13. Gilron I, Bailey JM, Tu D, Holden RR, Jackson AC, Houlden RL. Nortriptyline and gabapentin, alone and in combination for neuropathic pain: a double-blind, randomised controlled crossover trial. Lancet 2009;374(9697):1252-1261

14. Endo Pharmaceuticals. Lidoderm prescribing information. Available at: http://www.lidoderm.com/hcp/default.aspx. Accessed June 30, 2010
15. Eisenberg E, McNicol E, Carr DB. Opioids for neuropathic pain. Cochrane Database Syst Rev 2006;3:CD006146

16. Hollingshead J, Dühmke RM, Cornblath DR. Tramadol for neuropathic pain. Cochrane Database Syst Rev 2006;3: CD003726

17. Neuroges X. Qutenza prescribing information. Available at: http://www.qutenza.com/hcp/. Accessed June 30, 2010

18. Otto M, Bach FW, Jensen TS, Brøsen K, Sindrup SH. Escitalopram in painful polyneuropathy: a randomized, placebo-controlled, cross-over trial. Pain 2008;139(2):275283

19. Semenchuk MR, Sherman S, Davis B. Double-blind, randomized trial of bupropion SR for the treatment of neuropathic pain. Neurology 2001;57(9):1583-1588

20. GSK. Lamictal prescribing information. 2010. Available at: https://www.gsksource.com/gskprm/en/US/adirect/gskprm? cmd $=$ ProductDetailPage\&product_id=1244170588800\& featureKey $=600578$. Accessed June 30, 2010

21. Wiffen PJ, Rees J. Lamotrigine for acute and chronic pain. Cochrane Database Syst Rev 2007;(2):CD006044

22. Vinik AI, Tuchman M, Safirstein B, et al. Lamotrigine for treatment of pain associated with diabetic neuropathy: results of two randomized, double-blind, placebo-controlled studies. Pain 2007;128(1-2):169-179

23. Jose VM, Bhansali A, Hota D, Pandhi P. Randomized double-blind study comparing the efficacy and safety of lamotrigine and amitriptyline in painful diabetic neuropathy. Diabet Med 2007;24(4):377-383

24. Silver M, Blum D, Grainger J, Hammer AE, Quessy S. Double-blind, placebo-controlled trial of lamotrigine in combination with other medications for neuropathic pain. J Pain Symptom Manage 2007;34(4):446-454

25. UCB. Vimpat prescribing information. Available at: http:// www.vimpat.com/. Accessed June 30, 2010

26. Shaibani A, Fares S, Selam JL, et al. Lacosamide in painful diabetic neuropathy: an 18-week double-blind placebo-controlled trial. J Pain 2009;10(8):818-828

27. Wiffen PJ, McQuay HJ, Moore RA. Carbamazepine for acute and chronic pain. Cochrane Database Syst Rev 2005; (3):CD005451

28. Grosskopf J, Mazzola J, Wan Y, Hopwood M. A randomized, placebo-controlled study of oxcarbazepine in painful diabetic neuropathy. Acta Neurol Scand 2006;114(3):177-180

29. Dworkin RH, O'Connor AB, Audette J, et al. Recommendations for the pharmacological management of neuropathic pain: an overview and literature update. Mayo Clin Proc 2010;85(3, Suppl):S3-S14

30. Yuan RY, Sheu JJ, Yu JM, et al. Botulinum toxin for diabetic neuropathic pain: a randomized double-blind crossover trial. Neurology 2009;72(17):1473-1478

31. Challapalli V, Tremont-Lukats IW, McNicol ED, Lau J, Carr DB. Systemic administration of local anesthetic agents to relieve neuropathic pain. Cochrane Database Syst Rev 2005;(4):CD003345

32. Rogers M, Rasheed A, Moradimehr A, Baumrucker SJ. Memantine (Namenda) for neuropathic pain. Am J Hosp Palliat Care 2009;26(1):57-59

33. Thisted RA, Klaff L, Schwartz SL, et al. Dextromethorphan and quinidine in adult patients with uncontrolled painful diabetic peripheral neuropathy: a 29-day, multicenter, openlabel, dose-escalation study. Clin Ther 2006;28(10):16071618 